\title{
Intraoperative blood vessel detection and quantification: a Monte Carlo study
}

\author{
Amal Chaturvedi \\ Shetha A. Shukair \\ Mayank Vijayvergia \\ Paul Le Rolland \\ Jonathan W. Gunn \\ Hariharan Subramanian
}




\title{
Intraoperative blood vessel detection and quantification: a Monte Carlo study
}

\author{
Amal Chaturvedi, ${ }^{*}$ Shetha A. Shukair, Mayank Vijayvergia, Paul Le Rolland, Jonathan W. Gunn, and \\ Hariharan Subramanian \\ Briteseed LLC, Chicago, Illinois, United States
}

\begin{abstract}
With better surgical outcomes, quicker recovery times, decreased postoperative pain, and reduced scarring at the surgical site, the application of minimally invasive surgery (MIS) has gained a lot of prominence in the last 30 years. This change in surgical practice has taken away the ability of a surgeon to palpate for the presence of a blood vessel as would occur in an open procedure. They instead must rely on a laparoscopic video camera feed that unfortunately cannot detect the presence of a blood vessel hidden beneath tissue. In certain scenarios, a surgeon can accidentally cut a blood vessel, which can lead to severe, even fatal, complications. Here, we show that by adding a near-infrared LED and a photodiode onto the opposing jaws of laparoscopic graspers, blood vessels buried under tissue can be detected. We show the results of Monte Carlo simulations to support our theory that the blood vessels ranging from 3 to $6 \mathrm{~mm}$ buried under up to $1 \mathrm{~cm}$ of tissue can be detected and quantified. This technology could be added to already existing laparoscopic tools that have limited surface areas on the jaws to assist surgeons during MIS procedures. ๑ 2018 Society of Photo-Optical Instrumentation Engineers (SPIE) [DOI: 10.1117/1.JBO.23.6.067002]
\end{abstract}

Keywords: smart surgery; intelligent grasper; minimally invasive surgery; laparoscopic tools.

Paper 170817RR received Dec. 20, 2017; accepted for publication May 24, 2018; published online Jun. 13, 2018.

\section{Introduction}

The application of minimally invasive surgery (MIS) techniques has gained a lot of prominence in the last few decades. MIS techniques offer better surgical outcomes, quicker recovery times, decreased postoperative pain, and reduced scarring at the surgical site. ${ }^{1-4}$ However, with the increased adoption of MIS procedures, some challenges have emerged. Surgeons are unable to feel for the natural pulsation of blood flow within blood vessels making the identification of tissue-embedded blood vessels highly dependent on the experience of the surgeon. ${ }^{5}$ The challenges not only include the detection of hidden blood vessels but also the perioperative management of bleeding complications that arise. Inadvertent cuts into vasculature cause billions of dollars as nonreimbursable amount in hospital costs in the United States every year [assuming a $1 \%$ incidence rate (see Opitz et al.) and $\$ 210,000$ cost per incidence]. Studies also indicate that the most common cause for conversion during laparoscopic surgeries is excessive hemorrhage. ${ }^{6}$ Patients who suffer vascular injury during surgery may face an increased risk of developing an infection; decreased quality of life following discharge; or, in up to $32 \%$ of cases, death. $^{7}$ Moreover, those who do survive have an average added cost of care of hundreds of thousands of dollars due to corrective procedures and a hospital stay of multiple additional days. ${ }^{8,9}$ To assist surgeons in preventing these major complications, there is a need for advanced tools that can identify the presence of blood vessels that must be avoided or strategically divided in real time.

There are already existing modalities, such as ultrasound and fluorescence angiography, for intraoperative blood vessel

*Address all correspondence to: Amal Chaturvedi, E-mail: a.chaturvedi@ briteseed.com visualization, but they either lack the desired speed and resolution or are too bulky to be easily integrated into the surgical workflow. ${ }^{10-12}$ Near-infrared (NIR) imaging has been pursued in breast reconstruction and colon surgery to offer information about the microvasculature, but offers low sensitivity and requires expensive contrast agents. ${ }^{13}$ The main limitations of existing vascular detection systems include the inability to quantitate vessel metrics, added complexity during surgery, and cost. Importantly, advanced MIS tools are increasingly being miniaturized to increase maneuverability and allow access into tighter spaces during laparoscopic procedures. ${ }^{14,15}$ In addition, manufacturers have been advised by surgeons to increase multifunctional product offerings to further streamline costs and simplify procedures. $^{16,17}$

Briteseed has developed a technology that addresses these problems by exploiting the pulsatile light absorption characteristics of hemoglobin in blood; the technological basis of pulse oximetry, which is the standard of care for monitoring a patients' blood oxygen saturation. However, pulsatile light absorption has never been applied to intraoperative use for the real-time detection and analysis of vasculature. We have developed a light absorption-based technology that provides identification and characterization of vasculature during MIS procedures. Whereas pulse oximetry uses pulsatile light absorption properties of hemoglobin to provide blood oxygen saturation, our device uses pulsatile blood flow to identify and characterize blood vessels without the need of a contrast agent. We also created a prototype of the grasping device and added an LED and a photodetector on its opposing jaws. Importantly, the technology is simplified to contain a single LED and a photodetector on the opposing jaws in order to easily integrate them into MIS tools

$1083-3668 / 2018 / \$ 25.00$ @ 2018 SPIE 
with limited surface area such as a Maryland dissector (the jaws are about $22.4 \mathrm{~mm}$ long and have an average width of about $3 \mathrm{~mm}$ ), right angle dissector (jaws are $32 \mathrm{~mm}$ long and have an average width of about $3.75 \mathrm{~mm}$ ) or a laparoscopic stapler. These tools are typically used to dissect different tissues in various procedures, where it is critical to characterize the blood vessels.

We present the results of extensive Monte Carlo simulations to validate the theoretical foundation of this technology. We also present ex vivo experiment results to demonstrate feasibility of this technology using a prototype of the laparoscopic grasper. The prototype was first tested on blood vessel phantoms with and without porcine tissues and then on ex vivo porcine blood vessels. The results from these studies indicate that the system is able to quantify blood vessels between 3 and $6 \mathrm{~mm}$ in diameter buried under tissues as thick as $1 \mathrm{~cm}$. This quantification can be accomplished with minimal hardware components, allowing for the design of sensing-capable tools of nearly any size.

\section{Theory}

\subsection{Pulse Oximetry}

Pulse oximetry is a technique used to estimate the heart rate and the oxygen saturation of the blood noninvasively. In traditional settings, a pulse oximeter has an LED with two wavelengths, where one is in the visible range $(660 \mathrm{~nm})$ and the other is in NIR range $(910 \mathrm{~nm})$ on the one side and a photodetector on the other side. These wavelengths have different absorption properties based on the oxygenated and deoxygenated fractions of blood. The device is clipped onto the finger or ear of the person, where it shines light on that body part. The light is attenuated by the skin, underlying tissue and blood present in the capillaries. The attenuated light received by the photodetector is mainly related to the amount of tissue and the oxygen saturation of the blood. In time, the signal received by the sensor is sinusoidal because of the periodic expansion and contraction of the arterioles. The frequency of this signal gives the heart rate and the ratio of the normalized standard deviation of the absorption values of the two wavelengths gives the oxygen saturation of the blood. Pulse oximeters are widely used in different clinical settings and have validated the underlying principle of light absorption by periodic blood flow. The underlying technology behind our device is based on the pulsatile light absorption of arterial blood, similar to the technology behind pulse oximeters. ${ }^{18}$

\subsection{Prototype Design}

To test the technology in the ex vivo setup, we created a prototype of the smart grasper laparoscopic tool to. We added an LED (OSRAM SFH4441) with wavelength $940 \mathrm{~nm}$ and a photodetector (APMKorea DDN2090M) on the opposing jaws of the prototype (Fig. 1) to use the tool in the transmission mode. The prototype, which provides the initial proof of concept, utilizes infrared light to measure the presence and size of blood vessels. The LED and sensor are controlled by an Arduino Uno 8-bit AVR microcontroller board. The conditioning circuitry of the photodetector is made of a transimpedance amplifier and a noninverting amplifier of gain $K=6$ (both Texas Instruments OPA333). The anode of the photodetector was biased to $0.3 \mathrm{~V}$. The LED was driven with a pulse width modulation coming from the Arduino Uno at a frequency of $244 \mathrm{~Hz}$ through an Infineon IRLML0040 MOSFET transistor. It was then filtered

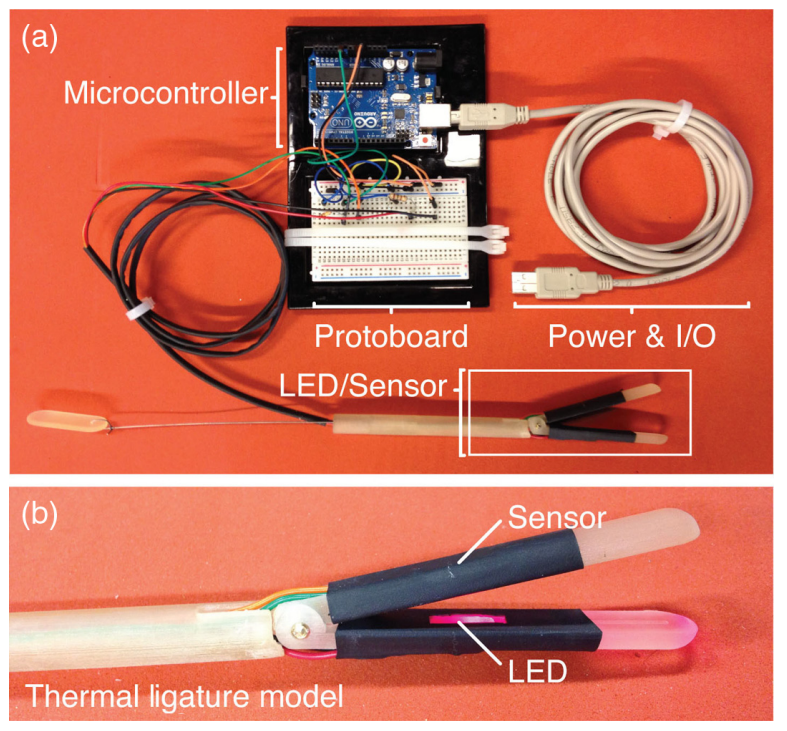

Fig. 1 The prototype of the smart grasper laparoscopic tool. One jaw of the prototype has an LED and the other has a photodetector on it.

through a passive RC low-pass filter (cut-off frequency $F_{c}=72 \mathrm{~Hz}, R=100 \Omega, C=22 \mu \mathrm{F}$ ) to prevent the pulsation of the LED to interfere with the readings of the photodetector. The light from the LED undergoes attenuation (absorption and scattering) that is dependent on the optical characteristics of the tissue and the blood it encounters. The sensor on the opposing jaw then receives this attenuated light. The received light is clearly a function of the size of the blood vessel it goes through. The output of the detector is fed to a computer through the USB cable. A custom GUI was developed in MATLAB ${ }^{\circledR}$ to collect, display, and analyze the signals.

\subsection{Principle of Operation}

As mentioned earlier, the underlying technology is based on the pulsatile absorption of transmitted light by the arterial blood. While pulse oximeters are used to measure blood oxygenation by quantifying light absorption at two different wavelengths (e.g., 660 and $910 \mathrm{~nm}$ ) in capillary beds, the same light absorption technology has not yet been applied to determine blood vessel presence or measure vessel diameter. In a general optics-based setup, the amount of light attenuation is used as a marker to understand the optical properties of the media. Beer-Lambert law states that the light gets attenuated exponentially with a linear increment in the distance it travels through an absorptive medium. More specifically, it states that if a light of a given intensity $\left(I_{0}\right)$ passes through a medium of a given length $(L)$, the intensity of the light received at the other end $(I)$ will be $I=I_{0} e^{-\alpha L}$ with attenuation coefficient $\alpha$. But in case of pulse oximetry or our setup, Beer-Lambert law is not valid as the light is also affected by the scattering caused by tissue (difference in the refractive index between cellular organelles and cellular fluid and between intracellular and extracellular fluids) and blood (difference in refractive index between red blood cells and plasma). ${ }^{19-21}$ For this reason, the Beer-Lambert law equation cannot be directly used to estimate the size of the blood vessel. A modified Beer-Lambert law was introduced for the scattering media ${ }^{22}$ and since its introduction has been a common technique used in the field of NIR tissue spectroscopy. It uses a mean path length of the detected photons instead of a direct 
source-detector distance. ${ }^{23}$ In our method, we show that the change in the signal amplitude relative to its average can be used to estimate the size of the absorbing medium (pulsing arteries).

As shown in Fig. 2, the transmitted light $(I)$ contains both a pulsatile component $\left(I_{\mathrm{AC}}\right)$ i.e., periodic light absorption by the blood flow within the vessel, and a baseline component $\left(I_{\mathrm{DC}}\right)$, i.e., static light absorption and scattering mainly by the surrounding tissues. To quantify the amount of change and the baseline of the signal, we take standard deviation and the average of the received signal, respectively. We call the standard deviation as the AC and the average as the DC, which are commonly used terms in the field of pulse oximetry. ${ }^{18}$ It is assumed that the larger the blood vessel, the higher the absorption and lower the DC will be; and since there is a higher amount of blood volume changes, the $\mathrm{AC}$, at the same LED brightness, will be smaller. In short, the fraction AC/DC should be inversely proportional to the blood vessel diameter. The system uses (a) Tool schematic

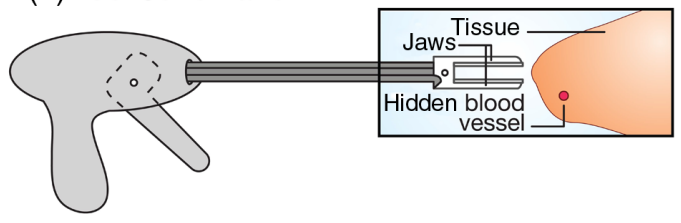

\section{(b) Signal with vessel}
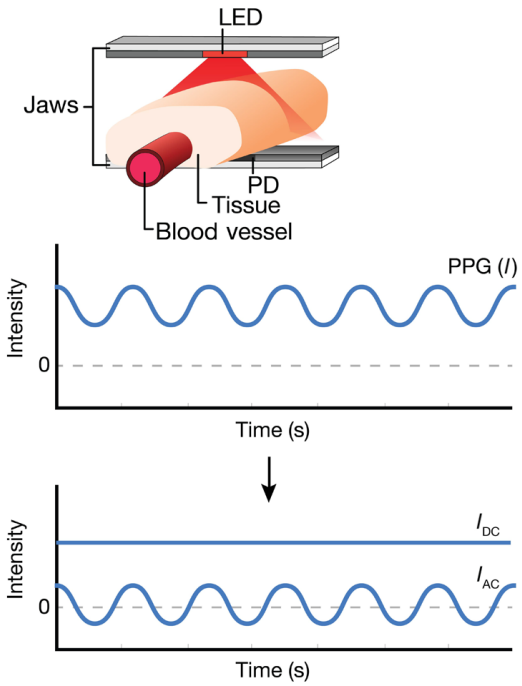

Fig. 2 The measurement setup used in this study. (a) An LED and a photodetector are added to the opposing jaws of a laparoscopic grasper. (b) The light received by the photodetector (PD) has both a pulsatile $\left(I_{A C}\right.$, created by periodic blood flow) as well as a baseline $\left(I_{D C}\right.$, mostly due to the tissue) component.

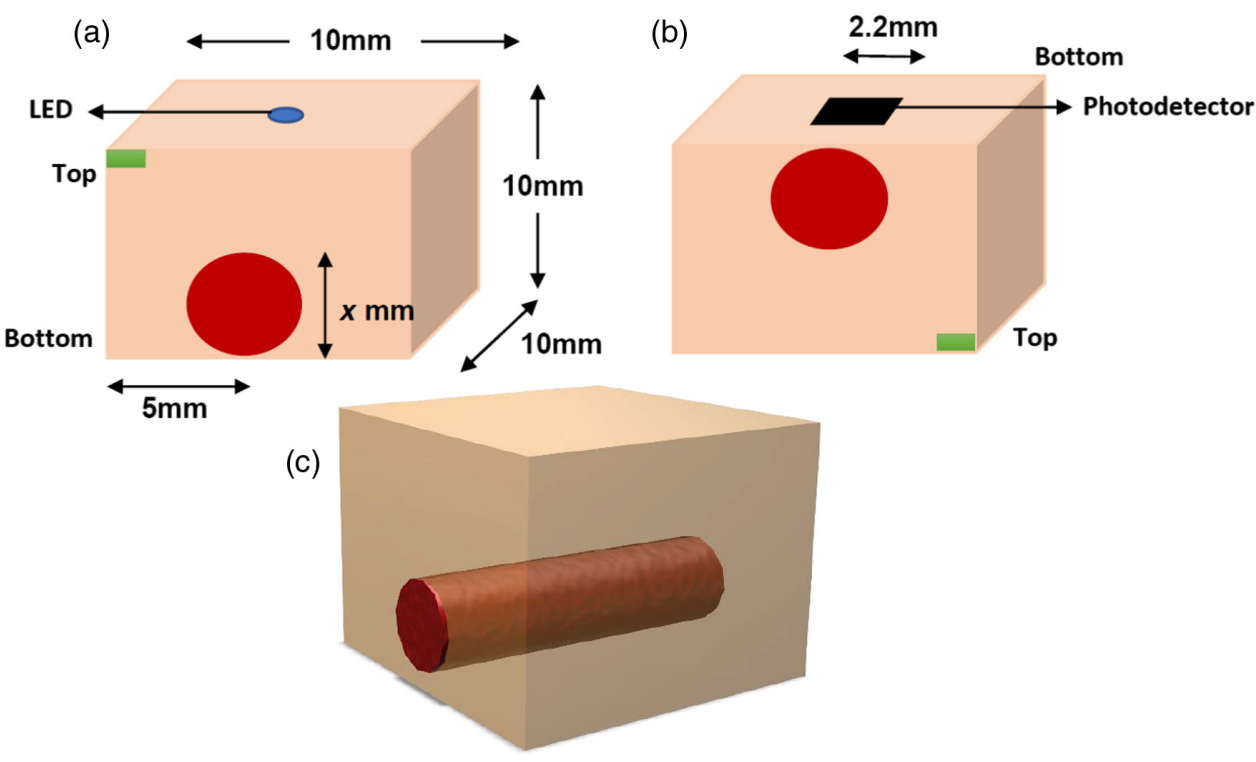

Fig. 3 A 3-D tissue block of size $10 \times 10 \times 10 \mathrm{~mm}^{3}$ was modeled. (a) An LED with wavelength $\lambda=940 \mathrm{~nm}$ was placed at center of the top surface of the block. The light from the LED undergoes some attenuation that is dependent on the optical characteristics of the tissue(s) and size of the blood vessel traversed by the photons. (b) The attenuated light that reaches the other side is recorded by the photodetector under the tissue block. The block is inverted upside down (b) to show the placement of the photodetector under the tissue block. The 3-D block in (c) shows how the cylindrical-shaped blood vessel runs through the tissue in a given depth. 
unique hardware and software to extract the $I_{A C}$ and $I_{D C}$ from the transmitted light and then analyze them to quantify the blood vessel size. Thus, our system is designed to selectively quantify the size of a blood vessel regardless of the type or thickness of tissue surrounding it. It is important to note here that the LED brightness plays a crucial role here. Too much or too little brightness can affect the resolution of $I_{A C}$ and for that reason, the brightness of the LED should be within an optimal range. For that purpose, we have developed a custom automated algorithm that updates the LED brightness until the signal received by the photodetector is five times above the noise floor. The noise floor is defined as the reading of the photodetector when the LED is completely turned off.

\subsection{Monte Carlo Simulation Validation}

Monte Carlo simulations serve as the benchmark in understanding the probabilistic behavior of light traversal inside a biological medium. Being simulations, they are not corrupted by realworld artifacts and therefore provide an unbiased understanding of the system under consideration. To work on the simulations, we created a 3-D model, where a cylindrical blood vessel was embedded within a thick block of tissue. An LED and a photodetector were placed at the top and bottom of the tissue block, respectively. The setup shown in Fig. 3 was used for these simulations.

We launched 150-million photons into the tissue block, orthogonal to the top surface of the block, with and without the blood vessel to simulate the irradiated LED light. The photons were launched in packets, and each packet was tracked individually as it moved through different voxels. The direction and weight of the photon packet were updated at each iteration depending on the scattering and absorption properties of that voxel. The tracking was discontinued once the weight of the packet went below a predefined threshold or it escaped from one of the surfaces of the block. If the photon escaped from the bottom surface within the coordinate region of the photodetector, computed using basic geometry and trigonometry, it was added to the count of the detected photons. In the end, the total number of detected photons formed the photodetector output for a one-time snapshot. This process (Fig. 4) was

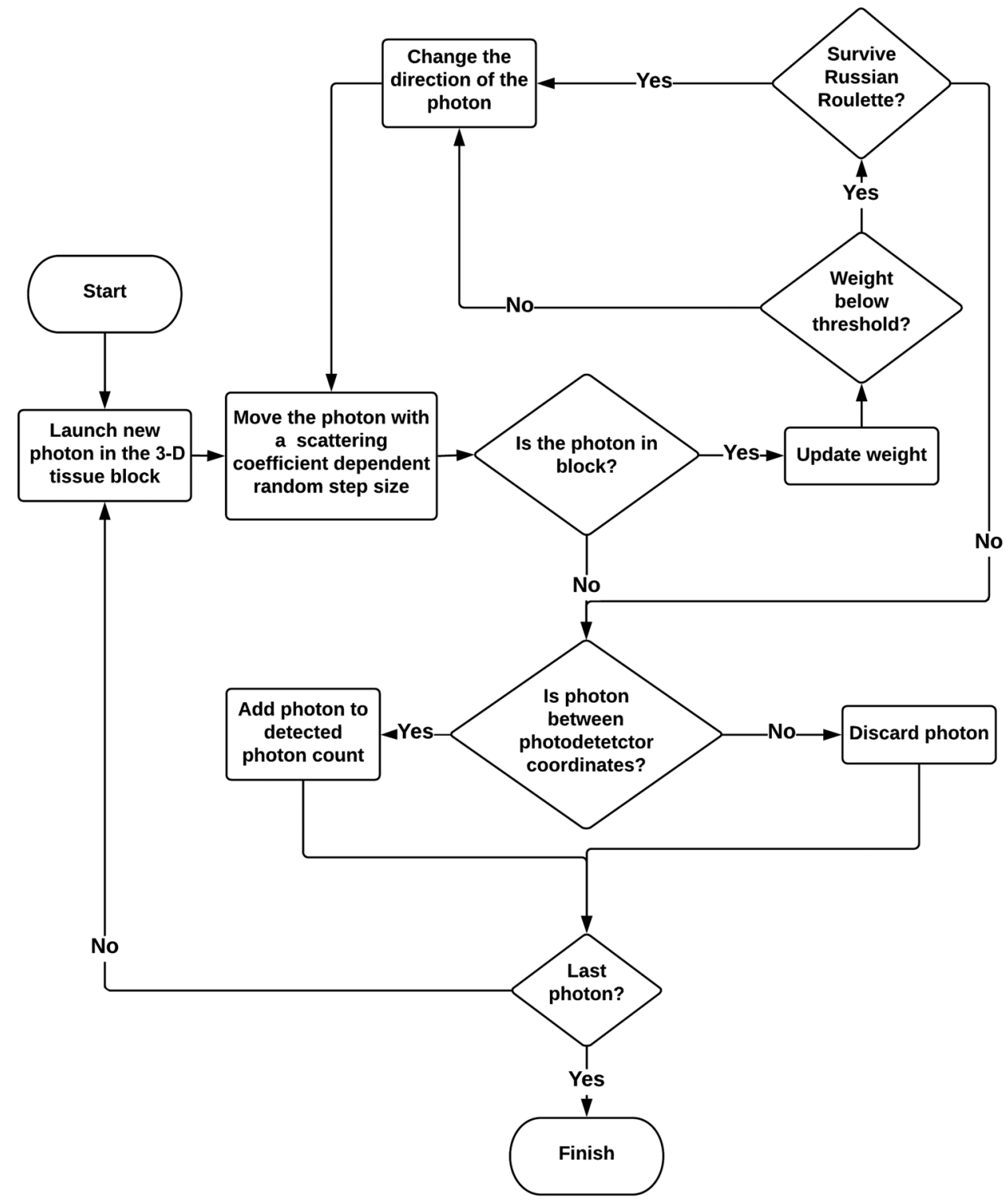

Fig. 4 Flowchart for the Monte Carlo simulations. 
repeated several times to create a signal received by the photodetector in time. The simulations were implemented in $\mathrm{C}$ based on the MCXYZ theory developed by Steven Jacques and Lihong Wang. ${ }^{24,25}$ The optical properties of the simulated blocks were specified in a Cartesian grid. Being cylindrical in shape, a blood vessel does not fit very well within a square grid, and hence there will be artifacts. To minimize these artifacts, a grid with sufficient resolution $(50 \mu \mathrm{m} \times 50 \mu \mathrm{m} \times 50 \mu \mathrm{m})$ was chosen such that the blood vessel is essentially round.

\section{Results and Discussion}

\subsection{Monte Carlo Simulation Results}

The Monte Carlo simulations were performed for blood vessels with outer diameter ranging between 1 and $6 \mathrm{~mm}$, with and without surrounding tissue. About 150-million photons were launched inside the tissue block. Adipose tissue $\left(\mu_{s}=122.9 \mathrm{~cm}^{-1}, \mu_{a}=0.13 \mathrm{~cm}^{-1}\right.$, and $g=0.9, n=1.4$ at $\lambda=940 \mathrm{~nm}^{26}$ ) was used for the initial analyses. An LED with wavelength $\lambda=940 \mathrm{~nm}$ was used as the light source. The detector used was a photodiode with dimensions of $2.2 \mathrm{~mm} \times 2.2 \mathrm{~mm}$. The blood vessel was placed on top of the photodetector. The optical properties for the blood used were $\mu_{s}=53.2 \mathrm{~cm}^{-1}, \mu_{\mathrm{a}}=6.62 \mathrm{~cm}^{-1}, g=0.9$, and $n=1.3$ at $\lambda=940 \mathrm{~nm}^{26}$ Each setup (a blood vessel of given diameter with or without tissue) was run 10 times to get one cycle of the periodic PPG signal. For each of these 10 simulations, the hemoglobin concentration was varied in a sinusoidal fashion to mimic the pulsatile flow of the blood through arteries. The following relation was used to create this periodic change in the hemoglobin concentration

$H(t)=\frac{A H_{0}}{100} \sin \left(\frac{2 \pi t}{T}\right)+H_{0} \quad \forall t \in[0, T)$,

where $H(t)$ is the hemoglobin concentration at discrete time $t, A$ is the amplitude of the sinusoidal variation, $H_{0}$ is the mean hemoglobin concentration $(g / L)$, and $T$ is the period. In our simulations, we used $H_{0}=150 \mathrm{~g} / L, A=10$, and $T=10$.

Figure 5 shows the PPGs of blood vessels that these simulations yielded. As can be seen in the figure, different blood vessels have a different rate of change of the pulsatile signals, which could be quantified and used as a marker to determine the size of the blood vessels. Figure 5(a) shows the normalized pulsatile signals recorded. These signals were normalized by the reading obtained from a simulation with same air block without a blood vessel. The signals were further normalized by their respective means to compare their shapes and amplitudes. Figure 5(b) shows these signals when normalized by the corresponding DCs.

As the amount of absorption depends on the amount of blood, which is directly related to the size of the blood vessels, the absorption is higher for blood vessels with larger diameters. As a result, the average light intensity received by the sensor for the larger blood vessels (DC) is lower. Normalizing the signals by their corresponding DCs shows that the pulse for the larger blood vessels is bigger indicating that the rate of change of blood flow is higher in the blood vessels with larger diameters. Plotting this normalized variation against the blood vessel diameter shows a clear relation between the two (Fig. 6). This relation could be used as a marker to estimate the size of the blood vessels.

The simulations were repeated for a setup, where the same blood vessels were buried deep under adipose tissue. The thickness of tissue surrounding the blood vessels was $1 \mathrm{~cm}$. The signals in Fig. 7(a) were obtained by normalizing the PPGs with the reading obtained from a simulation with same adipose tissue block without a blood vessel. These signals were further divided by their DCs to compare their shapes and amplitudes on the same scale. Figure 7(b) shows these signals when normalized by the corresponding DCs. Figures 5 and 7 show that the relative strengths of the signals received with and without tissue remain the same. They also demonstrate that the normalized signal variability of a blood vessel is directly proportional to its diameter.
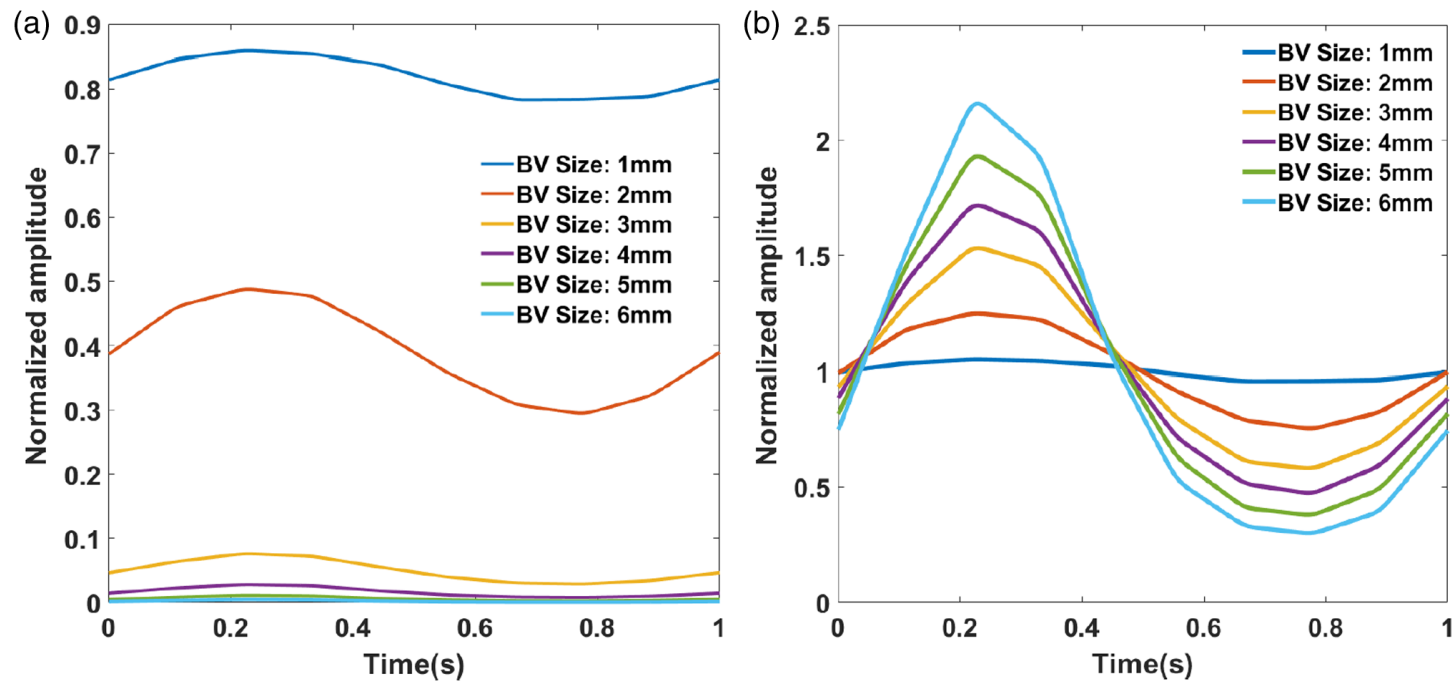

Fig. 5 The attenuated signal plotted over time is sinusoidal because of the pulsatile blood flow through the blood vessels. The absorption is higher for blood vessels with larger diameters as they contain more blood. (a) As a result the DC for the larger blood vessels is lower and the AC looks smaller because of the lower resolution. (b) Normalizing the signals by their DC values shows that the AC for the larger blood vessels is bigger. This suggests that the rate of change of blood flow is higher in blood vessels with larger diameters. 


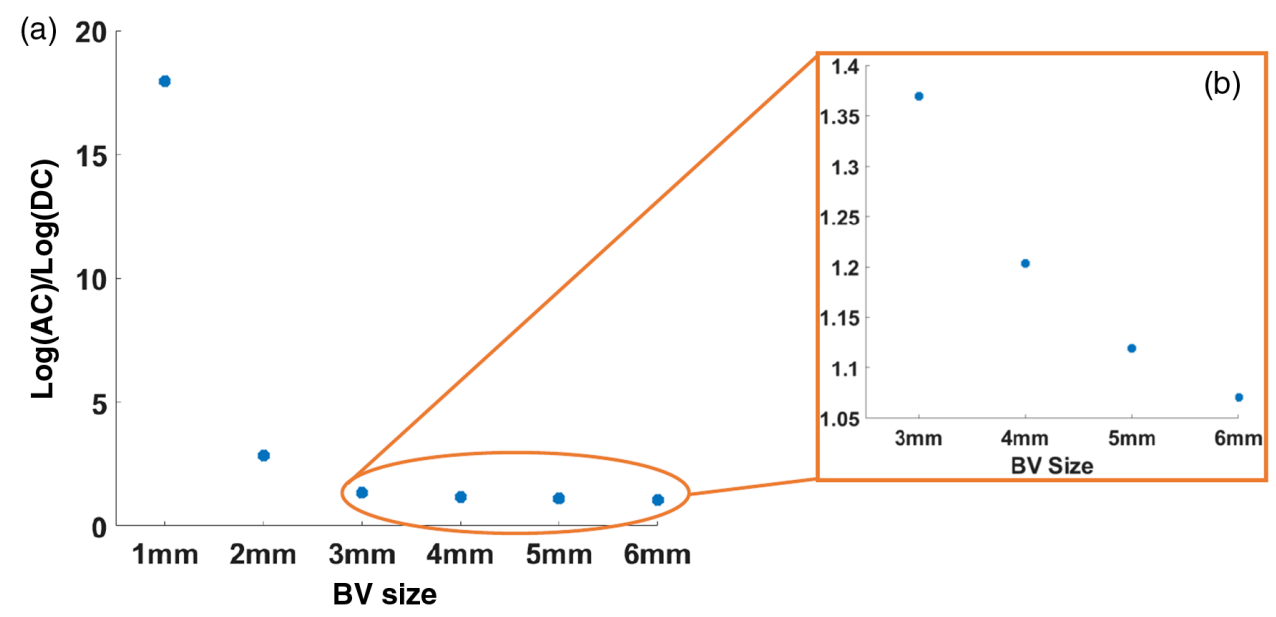

Fig. 6 (a) The plot between the ratio $\log (A C) / \log (D C)$ and the blood vessel diameter demonstrates a clear relationship between the two. This plot is for the skeletonized blood vessels, i.e., the blood vessels that do not have any tissue surrounding them. The AC is computed by taking the standard deviation of the signal, and the DC is the mean of the signal. (b) Zoomed in to show the ratio profile for blood vessels of size 3 to $6 \mathrm{~mm}$.
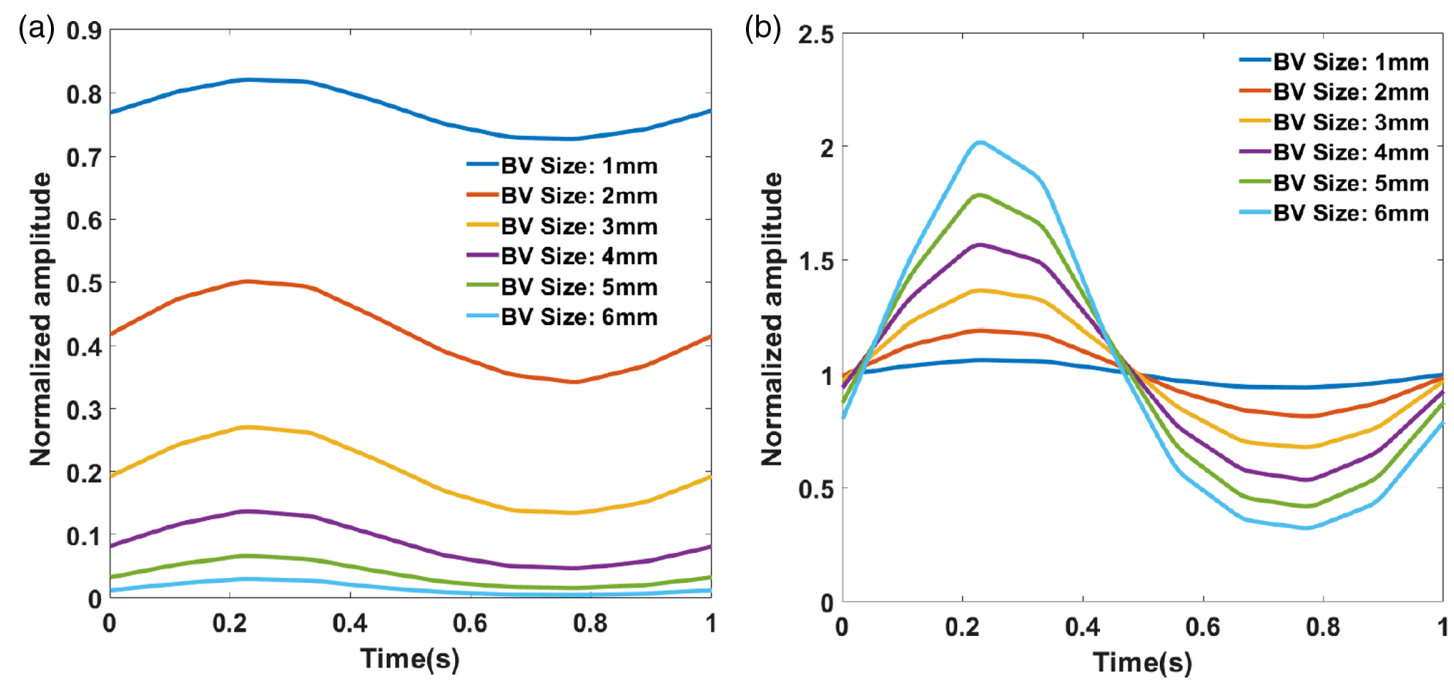

Fig. 7 The blood vessels were embedded in a 1-cm block of adipose tissue. (a) The signals for these simulations do not differ from those obtained using blood vessels alone [shown in Fig. 5(a)] when the PPGs are normalized with the reading obtained from a simulation with the adipose tissue block alone. (b) These signals were further normalized by their corresponding DCs to compare their shapes and amplitudes on the same scale, which highlights the same trend seen with blood vessel alone [shown in Fig. 5(b)].

Figure 8 shows that there is clear relationship between the ratio, $\log (\mathrm{AC}) / \log (\mathrm{DC})$, and the diameter of the blood vessel even if the vessel is buried under $1 \mathrm{~cm}$ of tissue.

\subsection{Results of Blood Vessel Phantom Experiments}

After validating the base theoretical concepts of the technology, we performed experiments with blood vessel mimicking tubes. The diameters of the tubes used varied from 3 to $6 \mathrm{~mm}$. The goal of this experiment was to determine whether the pulsatile component of the transmitted light $\left(I_{A C}\right)$ could be used to quantify different vessel sizes. We tested the system using pulsatile blood in optically clear tubing, which served as the in-vitro vessel phantom (control experiments showed no optical artifacts from the tubing material). The diameter of each size of tubing was provided by the manufacturer. A direct correlation between the $I_{A C}$ and vessel size was observed, indicating a strong proof of concept for blood vessel size quantification by the system. To get the pulsatile blood flow inside these tubes, we developed a test system that closely mimics the pulsatile flow found in arteries. This system employs a VPII submersible DC pump and an Arduino Uno (R3) microcontroller board to regulate both the volumetric flow rate and pulse rate. In order to measure the light transmittance through the phantom, an adjustable test rig was created for modifying the distance between the LED and detector pair. The pulsatile flow system was designed for operation between 40 and 80 cycles/min based on user input (to mimic the physiological heart beat) as well as to vary the flow rate (similar to changing cardiac output). In all the experiments discussed below, heparinized whole bovine blood was 


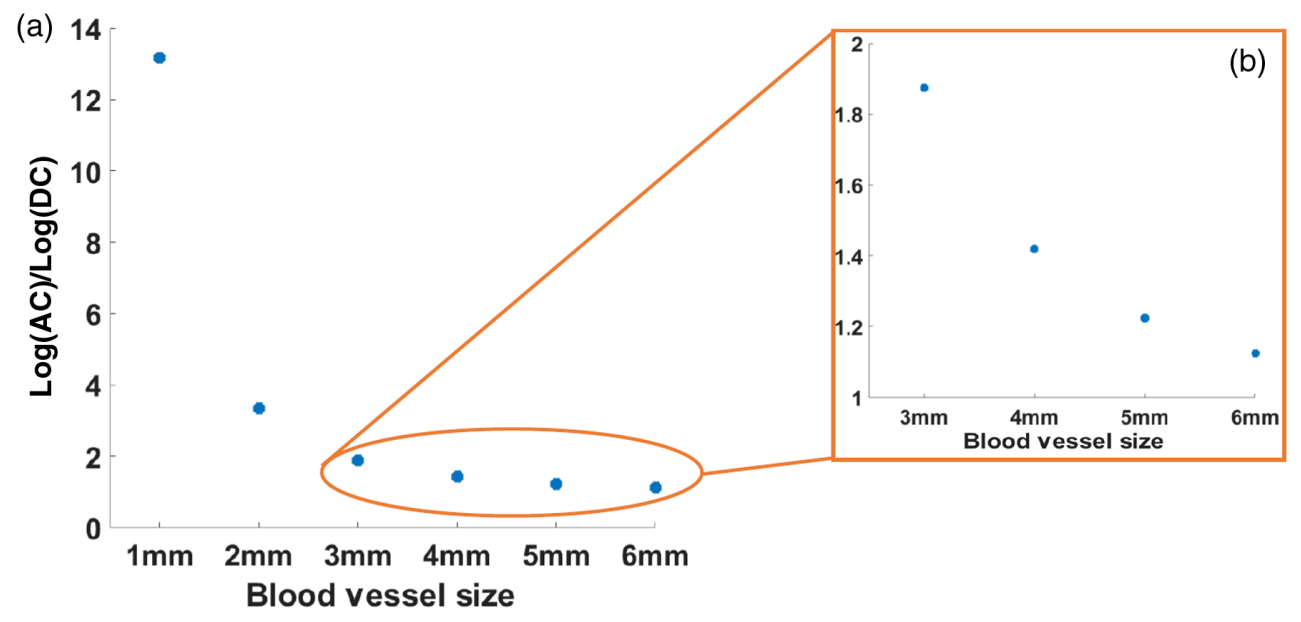

Fig. 8 (a) The $\log (A C) / \log (D C)$ versus blood vessel diameter plot brings up a similar trend as seen with the skeletonized blood vessels. This validates the hypothesis that even for blood vessels buried in tissue, the trend will remain the same as seen in the case of no tissue. (b) Zoomed in to show the ratio profile for blood vessels of size 3 to $6 \mathrm{~mm}$.

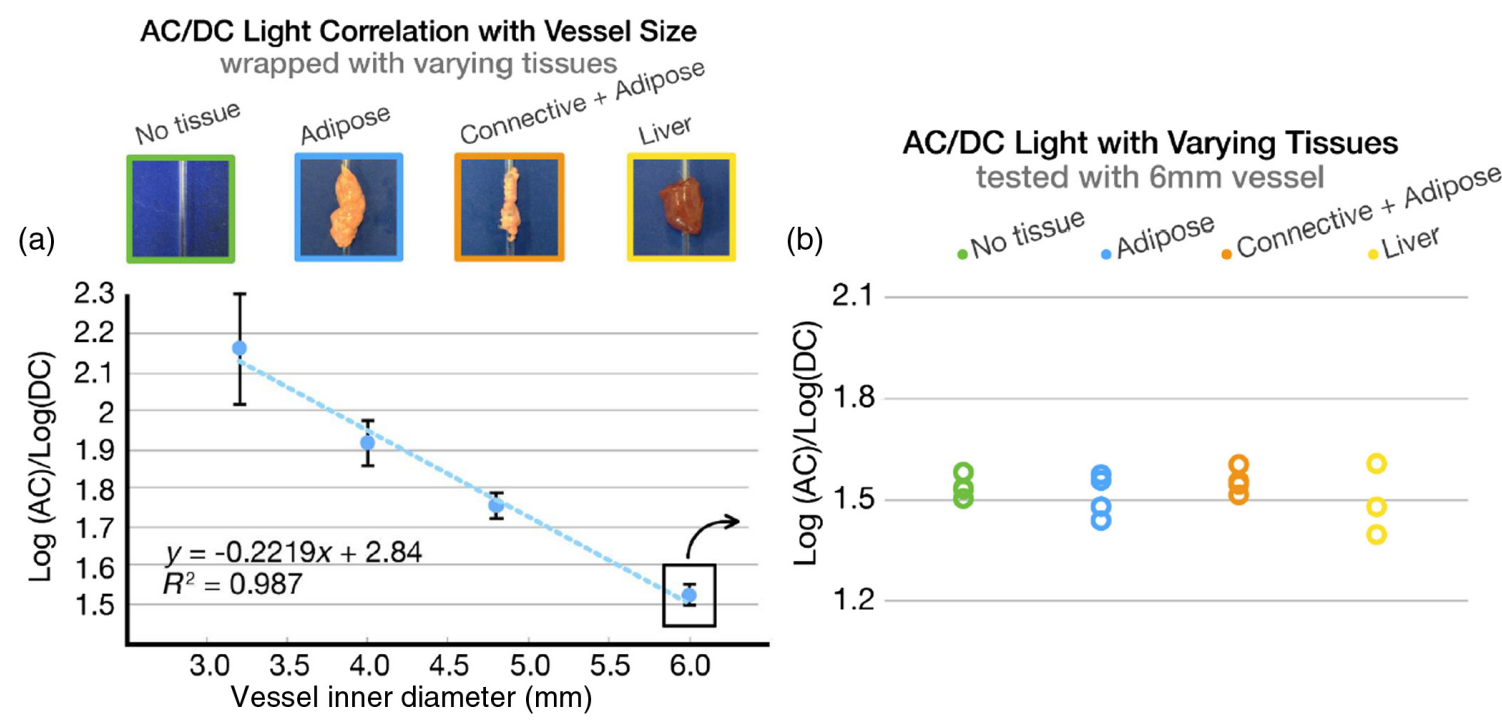

Fig. 9 (a) A linear correlation between the metric and the diameter of in vitro vessel phantoms (error bars are smaller than data symbols). (b) With various tissue types surrounding the vessel phantom, the metric was indicative of the actual vessel size. Each experiment was performed in triplicate.

pumped at 60 cycles/ min and the flow rate was maintained at $500 \mathrm{~mL} / \mathrm{min}$ (to mimic the blood flow of the hepatic artery). In addition, blood temperature was elevated to maintain physiological viscosity.

To check the effect of the added tissue on the signal characteristics, we wrapped the in-vitro vessel phantom (discussed above) with different types of porcine tissues. Sixty different samples were evaluated, including four vessel sizes, three tissue types, and one no-tissue control. In order to compensate for the scattering of light due to surrounding tissue, AC of the transmitted signal was normalized with DC and then correlated with vessel size. We found that regardless of tissue type or thickness, the $\log (\mathrm{AC}) / \log (\mathrm{DC})$ correlated very well $\left(R^{2}=0.987\right)$ with the diameter [Fig. 9(a)]. In addition, when looking at a single vessel size (e.g., 6-mm inner diameter), the different tissue wrapping

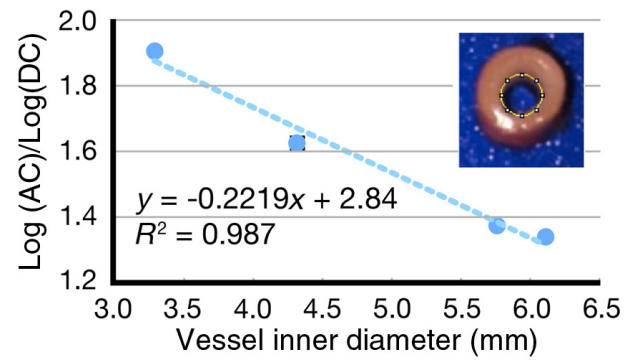

Fig. 10 A linear correlation between the metric and ex vivo porcine arteries (error bars are smaller than data symbols). Vessel diameter was quantified using NIH ImageJ (inset). 
samples had the same effective metric, indicating that the metric is independent of the surrounding tissue [Fig. 9(b)].

\subsection{Ex vivo Testing with Porcine Arteries}

We then used fresh porcine carotid arteries ( $n=5$ pigs) ex vivo in place of the PVC phantoms. The vessel size was obtained by quantifying the cross section of the vessels at the point of measurement using NIH ImageJ software (Fig. 10 inset). The results from the porcine blood vessel showed an excellent correlation $\left(R^{2}=0.987\right)$ between vessel size and the metric.

\section{Conclusion}

We have demonstrated that by putting an LED and a photodetector across a blood vessel, it is possible to detect the vessel and quantify its diameter with sub-mm resolution. We presented the results obtained with Monte Carlo simulations to validate the theoretical concepts behind the technology. After the theoretical proof, we performed the ex vivo experiments on PVC phantoms mimicking blood vessels of different diameter with and without added tissue. These experiments showed a similar trend as seen with the Monte Carlo simulations. To check the applicability of this technology in real blood vessels, we repeated the experiments with fresh porcine arteries and were able to detect and quantify 3 to $6 \mathrm{~mm}$ blood vessels with high resolution. In the future, we will be developing a handheld prototype that could be used for in vivo testing. These ex-vivo experiments combined with the theoretical proof provide a strong indication that such a technology could be used in real world settings. Importantly, a single LED and sensor pair is small enough to be integrated into existing laparoscopic tools (i.e., Maryland, Right Angle, Endo GIA, Echelon GST), where the jaws have extremely small surface area. The concept could be further translated to LED and sensor arrays to be integrated into larger tools such as laparoscopic graspers and dissectors (i.e., Hunter graspers, Debakey graspers, bowel graspers, and dolphin-tipped dissectors). It should be noted that the currently available imaging modalities do not provide such resolution at low costs.

\section{Disclosures}

Dr. Chaturvedi, Dr. Gunn, and Dr. Subramanian have a patent "System for Determining Vessel Size using Light Absorption" pending. In addition, Dr. Subramanian reports personal fees from Northwestern University, personal fees from NanoCytomics, outside the submitted work. Dr. Shukair, Mr. Vijayvergia, and Mr. Le Rolland have nothing to disclose.

\section{Acknowledgments}

We would like to acknowledge Dr. Andrew Radosevich for his help in the Monte Carlo modeling of blood vessels. We also thank Tom Bairaktaris of Park Packing Company (Chicago, IL) for donating porcine tissue blocks for the purposes of this research project.

\section{References}

1. H. L. Yeo et al., "Comparison of open, laparoscopic, and robotic colectomies using a large national database: outcomes and trends related to surgery center volume," Dis. Colon Rectum 59(6), 535-542 (2016).

2. S. L. Cohen, A. F. Vitonis, and J. I. Einarsson, "Updated hysterectomy surveillance and factors associated with minimally invasive hysterectomy," J. Soc. Laparoendosc. Surg. 18(3), e2014.00096 (2014).
3. A. Ejaz et al., "A comparison of open and minimally invasive surgery for hepatic and pancreatic resections using the nationwide inpatient sample," Surgery 156(3), 538-547 (2014).

4. D. Riviere et al., "Laparoscopic versus open distal pancreatectomy for pancreatic cancer," Cochrane Database Syst. Rev. 4, CD011391 (2016).

5. N. Crane et al., "Enhanced surgical imaging: laparoscopic vessel identification and assessment of tissue oxygenation," J. Am. Coll. Surg. 206, 1159-1166 (2008).

6. M. W. Rowley and J. S. Wolf Jr., "Risk factors for conversion to hand assisted laparoscopy or open surgery during laparoscopic renal surgery," J. Urol. 185(3), 940-944 (2011).

7. I. Opitz et al., "Bleeding remains a major complication during laparoscopic surgery: analysis of the salts database," Langenbeck's Arch. Surg. 390(2), 128-133 (2005).

8. M. E. Stokes et al., "Impact of bleeding-related complications and/or blood product transfusions on hospital costs in inpatient surgical patients," BMC Health Serv. Res. 11, 135-147 (2011).

9. A. R. Nandan et al., "The impact of major intraoperative adverse events on hospital readmissions," Am. J. Surg. 213(1), 10-17 (2017).

10. A. S. Sethi, S. M. Regan, and C. P. Sundaram, "The use of a Doppler ultrasound probe during vascular dissection in laparoscopic renal surgery," J. Endourol. 23(9), 1377-1382 (2009).

11. P. Newman and G. Rozycki, "The history of ultrasound," Surg. Clin. North Am. 78(2), 179-195 (1998).

12. J. L. Saumet et al., "Cutaneous laser-Doppler flowmetry: influence of underlying muscle blood flow," J. Appl. Physiol. 65(1), 478-481 (1988).

13. B. T. Lee et al., "Intraoperative near-infrared fluorescence imaging in perforator flap reconstruction: current research and early clinical experience," J. Reconstr. Microsurg. 26(1), 59-65 (2010).

14. P. P. Shadduck et al., "Mini-laparoscopy: instruments and economics," Surg. Technol. Int. 27, 59-64 (2015).

15. H. Feussner et al., "Developments in flexible endoscopic surgery: a review," Clin. Exp. Gastroenterol. 8, 31-42 (2015).

16. M. I. Frecker et al., "Laparoscopic multifunctional instruments: design and testing of initial prototypes," J. Soc. Laparoendosc. Surg. 9(1), 105-112 (2005).

17. D. J. Miller, C. A. Nelson, and D. Oleynikov, "Shortened or time and decreased patient risk through use of a modular surgical instrument with artificial intelligence," Surg. Endosc. 23(5), 1099-1105 (2009).

18. E. D. Chan, M. M. Chan, and M. M. Chan, "Pulse oximetry: understanding its basic principles facilitates appreciation of its limitations," Respir. Med. 107(6), 789-799 (2013).

19. M. Nitzan, "Pulse oximetry: fundamentals and technology update," Med. Devices 7, 231-239 (2013).

20. S. Matcher, "Signal quantification and localization in tissue near-infrared spectroscopy," in Handbook of Optical Biomedical Diagnostics, V. V. Tuchin, Ed., pp. 487-584, SPIE, Bellingham, Washington (2002).

21. S. Fantini and M. Francheschini, "Frequency domain-techniques for tissue spectroscopy and imaging," Chapter 7 in Handbook of Optical Biomedical Diagnostics, V. V. Tuchin, Ed., pp. 405-453, SPIE, Bellingham, Washington (2002).

22. D. T. Delpy et al., "Estimation of optical path length through tissue from direct time of flight measurements," Phys. Med. Biol. 33, 1433-1442 (1988).

23. A. Sassaroli and S. Fantini, "Comment on the modified Beer-Lambert law for scattering media," Phys. Med. Biol. 49, N255-N257 (2004).

24. S. L. Jacques et al., "mcxyz.c," https://omlc.org/software/mc/mcxyz (6 January 2017).

25. S. L. Jacques, "Coupling 3D Monte Carlo light transport in optically heterogeneous tissues to photoacoustic signal generation," Photoacoustics 2(4), 137-142 (2014).

26. S. L. Jacques, "Optical properties of biological tissues: a review," Phys. Med. Biol. 58(11), R37-R61 (2013).

Amal Chaturvedi is the lead signal processing engineer at Briteseed. He received his $\mathrm{MS}$ and $\mathrm{PhD}$ degrees in electrical engineering from the University of Cincinnati in 2012 and 2014, respectively.

Shetha A. Shukair is the lead research scientist at Briteseed. She received her $\mathrm{PhD}$ in life and biomedical sciences from Northwestern University in 2012. She has coauthored over 10 peer-reviewed articles. 
Mayank Vijayvergia is the head of business development and operations at Briteseed. He received his MS degree in biomedical engineering from Northwestern University in 2014.

Paul Le Rolland is the lead hardware engineer at Briteseed. He received his MS degree in electrical engineering from Illinois Institute of Technology in 2014.

Jonathan W. Gunn is the CEO at Briteseed. He received his $\mathrm{PhD}$ in biomaterials engineering and nanotechnology from the University of Washington in 2008 and his JD from Northwestern University in 2013.
He has more than a decade of experience in the biomedical engineering field and has authored over 15 peer-reviewed articles in the biomedical engineering field.

Hariharan Subramanian is the VP of research and development at Briteseed. He received his MS degree in biomedical engineering from Texas A\&M University in 2004 and his $\mathrm{PhD}$ in biomedical engineering from Northwestern University in 2009. His work has led to significant advancements in the fields of optical sensing systems and modern optical microscopy. He has authored over 40 peer-reviewed articles. 